\title{
The Real Vector Spaces of Finite Sequences are Finite Dimensional
}

\author{
Yatsuka Nakamura \\ Shinshu University \\ Nagano, Japan
}

\author{
Artur Korniłowicz \\ Institute of Computer Science \\ University of Białystok \\ Sosnowa 64, 15-887 Białystok, Poland
}

Summary. In this paper we show the finite dimensionality of real linear spaces with their carriers equal $\mathcal{R}^{n}$. We also give the standard basis of such spaces. For the set $\mathcal{R}^{n}$ we introduce the concepts of linear manifold subsets and orthogonal subsets. The cardinality of orthonormal basis of discussed spaces is proved to equal $n$.

MML identifier: EUCLID_7, version: $\underline{7.11 .014 .117 .1046}$

The articles [32], [7], [11], [33], [9], [2], [8], [5], [31], [4], [6], [18], [13], [22], [20], [14], [1], [21], [29], [28], [26], [3], [23], [10], [12], [30], [19], [34], [16], [17], [25], $[15],[24]$, and $[27]$ provide the notation and terminology for this paper.

\section{Preliminaries}

We use the following convention: $i, j, n$ are elements of $\mathbb{N}, z, B_{0}$ are sets, and $f, x_{0}$ are real-valued finite sequences.

Next we state several propositions:

(1) For all functions $f, g$ holds $\operatorname{dom}(f \cdot g)=\operatorname{dom} g \cap g^{-1}(\operatorname{dom} f)$.

(2) For every binary relation $R$ and for every set $Y$ such that $\operatorname{rng} R \subseteq Y$ holds $R^{-1}(Y)=\operatorname{dom} R$. 
(3) Let $X$ be a set, $Y$ be a non empty set, and $f$ be a function from $X$ into $Y$. If $f$ is bijective, then $\overline{\bar{X}}=\overline{\bar{Y}}$.

(4) $\langle z\rangle \cdot\langle 1\rangle=\langle z\rangle$.

(5) For every element $x$ of $\mathcal{R}^{0}$ holds $x=\varepsilon_{\mathbb{R}}$.

(6) For all elements $a, b, c$ of $\mathcal{R}^{n}$ holds $(a-b)+c+b=a+c$.

Let $f_{1}, f_{2}$ be finite sequences. One can verify that $\left\langle f_{1}, f_{2}\right\rangle$ is finite sequencelike.

Let $D$ be a set and let $f_{1}, f_{2}$ be finite sequences of elements of $D$. Then $\left\langle f_{1}, f_{2}\right\rangle$ is a finite sequence of elements of $D \times D$.

Let $h$ be a real-valued finite sequence. Let us observe that $h$ is increasing if and only if:

(Def. 1) For every $i$ such that $1 \leq i<\operatorname{len} h$ holds $h(i)<h(i+1)$.

One can prove the following four propositions:

(7) Let $h$ be a real-valued finite sequence. Suppose $h$ is increasing. Let given $i, j$. If $i<j$ and $1 \leq i$ and $j \leq \operatorname{len} h$, then $h(i)<h(j)$.

(8) Let $h$ be a real-valued finite sequence. Suppose $h$ is increasing. Let given $i, j$. If $i \leq j$ and $1 \leq i$ and $j \leq \operatorname{len} h$, then $h(i) \leq h(j)$.

(9) Let $h$ be a natural-valued finite sequence. Suppose $h$ is increasing. Let given $i$. If $1 \leq i \leq \operatorname{len} h$ and $1 \leq h(1)$, then $i \leq h(i)$.

(10) Let $V$ be a real linear space and $X$ be a subspace of $V$. Suppose $V$ is strict and $X$ is strict and the carrier of $X=$ the carrier of $V$. Then $X=V$.

Let $D$ be a set, let $F$ be a finite sequence of elements of $D$, and let $h$ be a permutation of $\operatorname{dom} F$. The functor $F \circ h$ yields a finite sequence of elements of $D$ and is defined as follows:

(Def. 2) $F \circ h=F \cdot h$.

One can prove the following propositions:

(11) Let $D$ be a non empty set and $f$ be a finite sequence of elements of $D$. If $1 \leq i \leq \operatorname{len} f$ and $1 \leq j \leq \operatorname{len} f$, then $(\operatorname{Swap}(f, i, j))(i)=f(j)$ and $(\operatorname{Swap}(f, i, j))(j)=f(i)$.

(12) $\emptyset$ is a permutation of $\emptyset$.

(13) $\langle 1\rangle$ is a permutation of $\{1\}$.

(14) For every finite sequence $h$ of elements of $\mathbb{R}$ holds $h$ is one-to-one iff sort $_{\mathrm{a}} h$ is one-to-one.

(15) Let $h$ be a finite sequence of elements of $\mathbb{N}$. Suppose $h$ is one-to-one. Then there exists a permutation $h_{3}$ of $\operatorname{dom} h$ and there exists a finite sequence $h_{2}$ of elements of $\mathbb{N}$ such that $h_{2}=h \cdot h_{3}$ and $h_{2}$ is increasing and $\operatorname{dom} h=\operatorname{dom} h_{2}$ and $\operatorname{rng} h=\operatorname{rng} h_{2}$. 


\section{Orthogonal Basis}

Let $B_{0}$ be a set. We say that $B_{0}$ is $\mathbb{R}$-orthogonal if and only if:

(Def. 3) For all real-valued finite sequences $x, y$ such that $x, y \in B_{0}$ and $x \neq y$ holds $|(x, y)|=0$.

Let us observe that every set which is empty is also $\mathbb{R}$-orthogonal.

We now state the proposition

(16) $B_{0}$ is $\mathbb{R}$-orthogonal if and only if for all points $x, y$ of $\mathcal{E}_{\mathrm{T}}^{n}$ such that $x$, $y \in B_{0}$ and $x \neq y$ holds $x, y$ are orthogonal.

Let $B_{0}$ be a set. We say that $B_{0}$ is $\mathbb{R}$-normal if and only if:

(Def. 4) For every real-valued finite sequence $x$ such that $x \in B_{0}$ holds $|x|=1$.

Let us observe that every set which is empty is also $\mathbb{R}$-normal.

Let us observe that there exists a set which is $\mathbb{R}$-normal.

Let $B_{0}, B_{1}$ be $\mathbb{R}$-normal sets. One can verify that $B_{0} \cup B_{1}$ is $\mathbb{R}$-normal.

One can prove the following propositions:

(17) If $|f|=1$, then $\{f\}$ is $\mathbb{R}$-normal.

(18) If $B_{0}$ is $\mathbb{R}$-normal and $\left|x_{0}\right|=1$, then $B_{0} \cup\left\{x_{0}\right\}$ is $\mathbb{R}$-normal.

Let $B_{0}$ be a set. We say that $B_{0}$ is $\mathbb{R}$-orthonormal if and only if:

(Def. 5) $\quad B_{0}$ is $\mathbb{R}$-orthogonal and $\mathbb{R}$-normal.

Let us note that every set which is $\mathbb{R}$-orthonormal is also $\mathbb{R}$-orthogonal and $\mathbb{R}$ normal and every set which is $\mathbb{R}$-orthogonal and $\mathbb{R}$-normal is also $\mathbb{R}$-orthonormal.

Let us observe that $\{\langle 1\rangle\}$ is $\mathbb{R}$-orthonormal.

Let us observe that there exists a set which is $\mathbb{R}$-orthonormal and non empty.

Let us consider $n$. One can verify that there exists a subset of $\mathcal{R}^{n}$ which is $\mathbb{R}$-orthonormal.

Let us consider $n$ and let $B_{0}$ be a subset of $\mathcal{R}^{n}$. We say that $B_{0}$ is complete if and only if:

(Def. 6) For every $\mathbb{R}$-orthonormal subset $B$ of $\mathcal{R}^{n}$ such that $B_{0} \subseteq B$ holds $B=B_{0}$.

Let $n$ be an element of $\mathbb{N}$ and let $B_{0}$ be a subset of $\mathcal{R}^{n}$. We say that $B_{0}$ is orthogonal basis if and only if:

(Def. 7) $\quad B_{0}$ is $\mathbb{R}$-orthonormal and complete.

Let us consider $n$. One can verify that every subset of $\mathcal{R}^{n}$ which is orthogonal basis is also $\mathbb{R}$-orthonormal and complete and every subset of $\mathcal{R}^{n}$ which is $\mathbb{R}$ orthonormal and complete is also orthogonal basis.

The following propositions are true:

(19) For every subset $B_{0}$ of $\mathcal{R}^{0}$ such that $B_{0}$ is orthogonal basis holds $B_{0}=\emptyset$. 
(20) Let $B_{0}$ be a subset of $\mathcal{R}^{n}$ and $y$ be an element of $\mathcal{R}^{n}$. Suppose $B_{0}$ is orthogonal basis and for every element $x$ of $\mathcal{R}^{n}$ such that $x \in B_{0}$ holds $|(x, y)|=0$. Then $y=\langle\underbrace{0, \ldots, 0}_{n}\rangle$.

\section{LiNEAR MANIFOLDS}

Let us consider $n$ and let $X$ be a subset of $\mathcal{R}^{n}$. We say that $X$ is linear manifold if and only if:

(Def. 8) For all elements $x, y$ of $\mathcal{R}^{n}$ and for all elements $a, b$ of $\mathbb{R}$ such that $x$, $y \in X$ holds $a \cdot x+b \cdot y \in X$.

Let us consider $n$. Observe that $\Omega_{\mathcal{R}^{n}}$ is linear manifold.

The following proposition is true

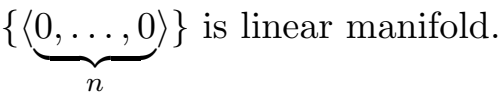

Let us consider $n$. Observe that $\{\langle\underbrace{0, \ldots, 0}_{n}\rangle$ is linear manifold.

Let us consider $n$ and let $X$ be a subset of $\mathcal{R}^{n}$. The linear span of $X$ yielding a subset of $\mathcal{R}^{n}$ is defined by:

(Def. 9) The linear span of $X=\bigcap\left\{Y \subseteq \mathcal{R}^{n}: Y\right.$ is linear manifold $\left.\wedge X \subseteq Y\right\}$.

Let us consider $n$ and let $X$ be a subset of $\mathcal{R}^{n}$. Observe that the linear span of $X$ is linear manifold.

Let us consider $n$ and let $f$ be a finite sequence of elements of $\mathcal{R}^{n}$. The functor $\sum f$ yielding an element of $\mathcal{R}^{n}$ is defined as follows:

(Def. 10)(i) There exists a finite sequence $g$ of elements of $\mathcal{R}^{n}$ such that len $f=$ len $g$ and $f(1)=g(1)$ and for every natural number $i$ such that $1 \leq i<$ len $f$ holds $g(i+1)=g_{i}+f_{i+1}$ and $\sum f=g(\operatorname{len} f)$ if len $f>0$,

(ii) $\sum f=\langle\underbrace{0, \ldots, 0}_{n}\rangle$, otherwise.

Let $n$ be a natural number and let $f$ be a finite sequence of elements of $\mathcal{R}^{n}$. The functor accum $f$ yields a finite sequence of elements of $\mathcal{R}^{n}$ and is defined as follows:

(Def. 11) len $f=$ len accum $f$ and $f(1)=(\operatorname{accum} f)(1)$ and for every natural number $i$ such that $1 \leq i<\operatorname{len} f$ holds $(\operatorname{accum} f)(i+1)=(\operatorname{accum} f)_{i}+f_{i+1}$.

We now state several propositions:

(22) For every finite sequence $f$ of elements of $\mathcal{R}^{n}$ such that len $f>0$ holds $(\operatorname{accum} f)(\operatorname{len} f)=\sum f$.

(23) For all finite sequences $F, F_{2}$ of elements of $\mathcal{R}^{n}$ and for every permutation $h$ of $\operatorname{dom} F$ such that $F_{2}=F \circ h$ holds $\sum F_{2}=\sum F$.

(24) For every element $k$ of $\mathbb{N}$ holds $\sum k \mapsto\langle\underbrace{0, \ldots, 0}_{n}\rangle=\langle\underbrace{0, \ldots, 0}_{n}\rangle$. 
(25) Let $g$ be a finite sequence of elements of $\mathcal{R}^{n}, h$ be a finite sequence of elements of $\mathbb{N}$, and $F$ be a finite sequence of elements of $\mathcal{R}^{n}$. Suppose $h$ is increasing and $\operatorname{rng} h \subseteq \operatorname{dom} g$ and $F=g \cdot h$ and for every element $i$ of $\mathbb{N}$ such that $i \in \operatorname{dom} g$ and $i \notin \operatorname{rng} h$ holds $g(i)=\langle\underbrace{0, \ldots, 0}_{n}\rangle$. Then $\sum g=\sum F$.

(26) Let $g$ be a finite sequence of elements of $\mathcal{R}^{n}, h$ be a finite sequence of elements of $\mathbb{N}$, and $F$ be a finite sequence of elements of $\mathcal{R}^{n}$. Suppose $h$ is one-to-one and $\operatorname{rng} h \subseteq \operatorname{dom} g$ and $F=g \cdot h$ and for every element $i$ of $\mathbb{N}$ such that $i \in \operatorname{dom} g$ and $i \notin \operatorname{rng} h$ holds $g(i)=\langle\underbrace{0, \ldots, 0}_{n}\rangle$. Then $\sum g=\sum F$

\section{Standard Basis}

Let us consider $n, i$. Then the base finite sequence of $n$ and $i$ is an element of $\mathcal{R}^{n}$.

The following propositions are true:

(27) Let $i_{1}, i_{2}$ be elements of $\mathbb{N}$. Suppose that

(i) $1 \leq i_{1}$,

(ii) $i_{1} \leq n$,

(iii) $1 \leq i_{2}$

(iv) $i_{2} \leq n$, and

(v) the base finite sequence of $n$ and $i_{1}=$ the base finite sequence of $n$ and $i_{2}$.

Then $i_{1}=i_{2}$.

(28) ${ }^{2}$ (the base finite sequence of $n$ and $i$ ) $=$ the base finite sequence of $n$ and $i$.

(29) If $1 \leq i \leq n$, then $\sum$ the base finite sequence of $n$ and $i=1$.

(30) If $1 \leq i \leq n$, then $\mid$ the base finite sequence of $n$ and $i \mid=1$.

(31) Suppose $1 \leq i \leq n$ and $1 \leq j \leq n$ and $i \neq j$. Then |(the base finite sequence of $n$ and $i$, the base finite sequence of $n$ and $j) \mid=0$.

(32) For every element $x$ of $\mathcal{R}^{n}$ such that $1 \leq i \leq n$ holds $\mid(x$, the base finite sequence of $n$ and $i) \mid=x(i)$.

Let us consider $n$ and let $x_{0}$ be an element of $\mathcal{R}^{n}$. The functor ProjFinSeq $x_{0}$ yields a finite sequence of elements of $\mathcal{R}^{n}$ and is defined by the conditions (Def. 12).

(Def. 12)(i) len ProjFinSeq $x_{0}=n$, and

(ii) for every $i$ such that $1 \leq i \leq n$ holds (ProjFinSeq $\left.x_{0}\right)(i)=\mid\left(x_{0}\right.$, the base finite sequence of $n$ and $i) \mid \cdot$ the base finite sequence of $n$ and $i$. 
The following proposition is true

(33) For every element $x_{0}$ of $\mathcal{R}^{n}$ holds $x_{0}=\sum$ ProjFinSeq $x_{0}$.

Let us consider $n$. The functor $\mathbb{R N}$-Base $n$ yields a subset of $\mathcal{R}^{n}$ and is defined by:

(Def. 13) $\mathbb{R N}$-Base $n=\{$ the base finite sequence of $n$ and $i$; $i$ ranges over elements of $\mathbb{N}: 1 \leq i \wedge i \leq n\}$.

Next we state the proposition

(34) For every non zero element $n$ of $\mathbb{N}$ holds $\mathbb{R}$-Base $n \neq \emptyset$.

Let us mention that $\mathbb{R N}$-Base 0 is empty.

Let $n$ be a non zero element of $\mathbb{N}$. Note that $\mathbb{R N}$-Base $n$ is non empty.

Let us consider $n$. Observe that $\mathbb{R N}$-Base $n$ is orthogonal basis.

Let us consider $n$. Observe that there exists a subset of $\mathcal{R}^{n}$ which is orthogonal basis.

Let us consider $n$. An orthogonal basis of $n$ is an orthogonal basis subset of $\mathcal{R}^{n}$.

Let $n$ be a non zero element of $\mathbb{N}$. Observe that every orthogonal basis of $n$ is non empty.

\section{Finite Real Unitary Spaces and Finite Real Linear Spaces}

Let $n$ be an element of $\mathbb{N}$. Observe that $\left\langle\mathcal{E}^{n},(\cdot \mid \cdot)\right\rangle$ is constituted finite sequences. Let $n$ be an element of $\mathbb{N}$. One can check that every element of $\left\langle\mathcal{E}^{n},(\cdot \mid \cdot)\right\rangle$ is real-valued.

Let $n$ be an element of $\mathbb{N}$, let $x, y$ be vectors of $\left\langle\mathcal{E}^{n},(\cdot \mid \cdot)\right\rangle$, and let $a, b$ be real-valued functions. One can verify that $x+y$ and $a+b$ can be identified when $x=a$ and $y=b$.

Let $n$ be an element of $\mathbb{N}$, let $x$ be a vector of $\left\langle\mathcal{E}^{n},(\cdot \mid \cdot)\right\rangle$, let $y$ be a realvalued function, and let $a, b$ be elements of $\mathbb{R}$. Observe that $a \cdot x$ and $b y$ can be identified when $a=b$ and $x=y$.

Let $n$ be an element of $\mathbb{N}$, let $x$ be a vector of $\left\langle\mathcal{E}^{n},(\cdot \mid \cdot)\right\rangle$, and let $a$ be a real-valued function. Observe that $-x$ and $-a$ can be identified when $x=a$.

Let $n$ be an element of $\mathbb{N}$, let $x, y$ be vectors of $\left\langle\mathcal{E}^{n},(\cdot \mid \cdot)\right\rangle$, and let $a, b$ be real-valued functions. One can check that $x-y$ and $a-b$ can be identified when $x=a$ and $y=b$. The following three propositions are true:

(35) Let $n$ be an element of $\mathbb{N}, x, y$ be elements of $\mathcal{R}^{n}$, and $u, v$ be points of $\left\langle\mathcal{E}^{n},(\cdot \mid \cdot)\right\rangle$. If $x=u$ and $y=v$, then $\otimes_{\mathcal{E}^{n}}(\langle u, v\rangle)=|(x, y)|$.

(36) Let $n, j$ be elements of $\mathbb{N}, F$ be a finite sequence of elements of the carrier of $\left\langle\mathcal{E}^{n},(\cdot \mid \cdot)\right\rangle, B_{2}$ be a subset of $\left\langle\mathcal{E}^{n},(\cdot \mid \cdot)\right\rangle, v_{0}$ be an element of $\left\langle\mathcal{E}^{n},(\cdot \mid \cdot)\right\rangle$, and $l$ be a linear combination of $B_{2}$. Suppose $F$ is one-to-one and $B_{2}$ is $\mathbb{R}$-orthogonal and $\operatorname{rng} F=$ the support of $l$ and $v_{0} \in B_{2}$ and $j \in \operatorname{dom}(l F)$ and $v_{0}=F(j)$. Then $\otimes_{\mathcal{E}^{n}}\left(\left\langle v_{0}, \sum l F\right\rangle\right)=\otimes_{\mathcal{E}^{n}}\left(\left\langle v_{0}, l\left(F_{j}\right) \cdot v_{0}\right\rangle\right)$. 
(37) Let $n$ be an element of $\mathbb{N}, f$ be a finite sequence of elements of $\mathcal{R}^{n}$, and $g$ be a finite sequence of elements of the carrier of $\left\langle\mathcal{E}^{n},(\cdot \mid \cdot)\right\rangle$. If $f=g$, then $\sum f=\sum g$.

Let $A$ be a set. Note that $\mathbb{R}_{\mathbb{R}}^{A}$ is constituted functions.

Let us consider $n$. Observe that $\mathbb{R}_{\mathbb{R}}^{\operatorname{Seg} n}$ is constituted finite sequences.

Let $A$ be a set. One can verify that every element of $\mathbb{R}_{\mathbb{R}}^{A}$ is real-valued.

Let $A$ be a set, let $x, y$ be vectors of $\mathbb{R}_{\mathbb{R}}^{A}$, and let $a, b$ be real-valued functions. Observe that $x+y$ and $a+b$ can be identified when $x=a$ and $y=b$.

Let $A$ be a set, let $x$ be a vector of $\mathbb{R}_{\mathbb{R}}^{A}$, let $y$ be a real-valued function, and let $a, b$ be elements of $\mathbb{R}$. Observe that $a \cdot x$ and $b y$ can be identified when $a=b$ and $x=y$.

Let $A$ be a set, let $x$ be a vector of $\mathbb{R}_{\mathbb{R}}^{A}$, and let $a$ be a real-valued function. One can check that $-x$ and $-a$ can be identified when $x=a$.

Let $A$ be a set, let $x, y$ be vectors of $\mathbb{R}_{\mathbb{R}}^{A}$, and let $a, b$ be real-valued functions. Observe that $x-y$ and $a-b$ can be identified when $x=a$ and $y=b$.

The following propositions are true:

(38) Let $X$ be a subspace of $\mathbb{R}_{\mathbb{R}}^{\operatorname{Seg} n}, x$ be an element of $\mathcal{R}^{n}$, and $a$ be a real number. If $x \in$ the carrier of $X$, then $a \cdot x \in$ the carrier of $X$.

(39) Let $X$ be a subspace of $\mathbb{R}_{\mathbb{R}}^{\operatorname{Seg} n}$ and $x, y$ be elements of $\mathcal{R}^{n}$. Suppose $x \in$ the carrier of $X$ and $y \in$ the carrier of $X$. Then $x+y \in$ the carrier of $X$.

(40) Let $X$ be a subspace of $\mathbb{R}_{\mathbb{R}}^{\operatorname{Seg} n}, x, y$ be elements of $\mathcal{R}^{n}$, and $a, b$ be real numbers. Suppose $x \in$ the carrier of $X$ and $y \in$ the carrier of $X$. Then $a \cdot x+b \cdot y \in$ the carrier of $X$.

(41) For all elements $x, y$ of $\mathcal{R}^{n}$ and for all points $u, v$ of $\mathbb{R}_{\mathbb{R}}^{\mathrm{Seg} n}$ such that $x=u$ and $y=v$ holds $\otimes_{\mathcal{E}^{n}}(\langle u, v\rangle)=|(x, y)|$.

(42) Let $F$ be a finite sequence of elements of the carrier of $\mathbb{R}_{\mathbb{R}}^{\operatorname{Seg} n}, B_{2}$ be a subset of $\mathbb{R}_{\mathbb{R}}^{\mathrm{Seg} n}, v_{0}$ be an element of $\mathbb{R}_{\mathbb{R}}^{\mathrm{Seg} n}$, and $l$ be a linear combination of $B_{2}$. Suppose $F$ is one-to-one and $B_{2}$ is $\mathbb{R}$-orthogonal and $\operatorname{rng} F=$ the support of $l$ and $v_{0} \in B_{2}$ and $j \in \operatorname{dom}(l F)$ and $v_{0}=F(j)$. Then $\otimes_{\mathcal{E}^{n}}\left(\left\langle v_{0}\right.\right.$, $\left.\left.\sum l F\right\rangle\right)=\otimes_{\mathcal{E}^{n}}\left(\left\langle v_{0}, l\left(F_{j}\right) \cdot v_{0}\right\rangle\right)$.

Let us consider $n$. Note that every subset of $\mathbb{R}_{\mathbb{R}}^{\operatorname{Seg} n}$ which is $\mathbb{R}$-orthonormal is also linearly independent.

Let $n$ be an element of $\mathbb{N}$. Note that every subset of $\left\langle\mathcal{E}^{n},(\cdot \mid \cdot)\right\rangle$ which is $\mathbb{R}$-orthonormal is also linearly independent. Next we state the proposition

(43) Let $B_{2}$ be a subset of $\mathbb{R}_{\mathbb{R}}^{\operatorname{Seg} n}, x, y$ be elements of $\mathcal{R}^{n}$, and $a$ be a real number. If $B_{2}$ is linearly independent and $x, y \in B_{2}$ and $y=a \cdot x$, then $x=y$. 


\section{Finite Dimensionality of the Spaces}

Let us consider $n$. One can check that $\mathbb{R N}$-Base $n$ is finite.

The following propositions are true:

(44) $\quad$ card $\mathbb{R N}$-Base $n=n$.

(45) Let $f$ be a finite sequence of elements of $\mathcal{R}^{n}$ and $g$ be a finite sequence of elements of the carrier of $\mathbb{R}_{\mathbb{R}}^{\operatorname{Seg} n}$. If $f=g$, then $\sum f=\sum g$.

(46) Let $x_{0}$ be an element of $\mathbb{R}_{\mathbb{R}}^{\mathrm{Seg} n}$ and $B$ be a subset of $\mathbb{R}_{\mathbb{R}}^{\mathrm{Seg} n}$. If $B=$ $\mathbb{R} N$-Base $n$, then there exists a linear combination $l$ of $B$ such that $x_{0}=$ $\sum l$.

(47) Let $n$ be an element of $\mathbb{N}, x_{0}$ be an element of $\left\langle\mathcal{E}^{n},(\cdot \mid \cdot)\right\rangle$, and $B$ be a subset of $\left\langle\mathcal{E}^{n},(\cdot \mid \cdot)\right\rangle$. If $B=\mathbb{R N}$-Base $n$, then there exists a linear combination $l$ of $B$ such that $x_{0}=\sum l$.

(48) For every subset $B$ of $\mathbb{R}_{\mathbb{R}}^{\operatorname{Seg} n}$ such that $B=\mathbb{R N}$-Base $n$ holds $B$ is a basis of $\mathbb{R}_{\mathbb{R}}^{\operatorname{Seg} n}$.

Let us consider $n$. Observe that $\mathbb{R}_{\mathbb{R}}^{\operatorname{Seg} n}$ is finite dimensional.

We now state several propositions:

(49) $\operatorname{dim}\left(\mathbb{R}_{\mathbb{R}}^{\operatorname{Seg} n}\right)=n$.

(50) For every subset $B$ of $\mathbb{R}_{\mathbb{R}}^{\operatorname{Seg} n}$ such that $B$ is a basis of $\mathbb{R}_{\mathbb{R}}^{\operatorname{Seg} n}$ holds $\overline{\bar{B}}=n$.

(51) $\emptyset$ is a basis of $\mathbb{R}_{\mathbb{R}}^{\text {Seg } 0}$.

(52) For every element $n$ of $\mathbb{N}$ holds $\mathbb{R} N-B$ ase $n$ is a basis of $\left\langle\mathcal{E}^{n},(\cdot \mid \cdot)\right\rangle$.

(53) Every orthogonal basis of $n$ is a basis of $\mathbb{R}_{\mathbb{R}}^{\operatorname{Seg} n}$.

Let $n$ be an element of $\mathbb{N}$. Note that $\left\langle\mathcal{E}^{n},(\cdot \mid \cdot)\right\rangle$ is finite dimensional.

We now state two propositions:

(54) For every element $n$ of $\mathbb{N}$ holds $\operatorname{dim}\left(\left\langle\mathcal{E}^{n},(\cdot \mid \cdot)\right\rangle\right)=n$.

(55) For every orthogonal basis $B$ of $n$ holds $\overline{\bar{B}}=n$.

\section{REFERENCES}

[1] Kanchun and Yatsuka Nakamura. The inner product of finite sequences and of points of $n$-dimensional topological space. Formalized Mathematics, 11(2):179-183, 2003.

[2] Grzegorz Bancerek. Cardinal numbers. Formalized Mathematics, 1(2):377-382, 1990.

[3] Grzegorz Bancerek. The fundamental properties of natural numbers. Formalized Mathematics, 1(1):41-46, 1990.

[4] Grzegorz Bancerek and Krzysztof Hryniewiecki. Segments of natural numbers and finite sequences. Formalized Mathematics, 1(1):107-114, 1990.

[5] Czesław Byliński. Basic functions and operations on functions. Formalized Mathematics, 1(1):245-254, 1990.

[6] Czesław Byliński. Finite sequences and tuples of elements of a non-empty sets. Formalized Mathematics, 1(3):529-536, 1990.

[7] Czesław Byliński. Functions and their basic properties. Formalized Mathematics, 1(1):55$65,1990$.

[8] Czesław Byliński. Functions from a set to a set. Formalized Mathematics, 1(1):153-164, 1990. 
[9] Czesław Byliński. Partial functions. Formalized Mathematics, 1(2):357-367, 1990.

[10] Czesław Byliński. Some basic properties of sets. Formalized Mathematics, 1(1):47-53, 1990.

[11] Czesław Byliński. The sum and product of finite sequences of real numbers. Formalized Mathematics, 1(4):661-668, 1990.

[12] Jing-Chao Chen. The Steinitz theorem and the dimension of a real linear space. Formalized Mathematics, 6(3):411-415, 1997.

[13] Agata Darmochwał. Finite sets. Formalized Mathematics, 1(1):165-167, 1990.

[14] Agata Darmochwał. The Euclidean space. Formalized Mathematics, 2(4):599-603, 1991.

[15] Noboru Endou, Takashi Mitsuishi, and Yasunari Shidama. Dimension of real unitary space. Formalized Mathematics, 11(1):23-28, 2003.

[16] Noboru Endou, Takashi Mitsuishi, and Yasunari Shidama. Linear combinations in real unitary space. Formalized Mathematics, 11(1):17-22, 2003.

[17] Noboru Endou and Yasunari Shidama. Completeness of the real Euclidean space. Formalized Mathematics, 13(4):577-580, 2005.

[18] Jarosław Kotowicz. Real sequences and basic operations on them. Formalized Mathematics, 1(2):269-272, 1990.

[19] Yatsuka Nakamura. Sorting operators for finite sequences. Formalized Mathematics, 12(1):1-4, 2004.

[20] Henryk Oryszczyszyn and Krzysztof Prażmowski. Real functions spaces. Formalized Mathematics, 1(3):555-561, 1990.

[21] Beata Padlewska. Families of sets. Formalized Mathematics, 1(1):147-152, 1990.

[22] Beata Padlewska and Agata Darmochwał. Topological spaces and continuous functions. Formalized Mathematics, 1(1):223-230, 1990.

[23] Nobuyuki Tamura and Yatsuka Nakamura. Determinant and inverse of matrices of real elements. Formalized Mathematics, 15(3):127-136, 2007, doi:10.2478/v10037-007-00014-7.

[24] Andrzej Trybulec. Binary operations applied to functions. Formalized Mathematics, $1(2): 329-334,1990$

[25] Andrzej Trybulec. Domains and their Cartesian products. Formalized Mathematics, 1(1):115-122, 1990

[26] Wojciech A. Trybulec. Basis of real linear space. Formalized Mathematics, 1(5):847-850, 1990.

[27] Wojciech A. Trybulec. Binary operations on finite sequences. Formalized Mathematics, 1(5):979-981, 1990

[28] Wojciech A. Trybulec. Linear combinations in real linear space. Formalized Mathematics, $1(3): 581-588,1990$.

[29] Wojciech A. Trybulec. Subspaces and cosets of subspaces in real linear space. Formalized Mathematics, 1(2):297-301, 1990.

[30] Wojciech A. Trybulec. Vectors in real linear space. Formalized Mathematics, 1(2):291-296, 1990.

[31] Zinaida Trybulec. Properties of subsets. Formalized Mathematics, 1(1):67-71, 1990.

[32] Edmund Woronowicz. Relations and their basic properties. Formalized Mathematics, 1(1):73-83, 1990.

[33] Edmund Woronowicz. Relations defined on sets. Formalized Mathematics, 1(1):181-186, 1990.

[34] Hiroshi Yamazaki, Yoshinori Fujisawa, and Yatsuka Nakamura. On replace function and swap function for finite sequences. Formalized Mathematics, 9(3):471-474, 2001. 